\title{
Electro-pneumatic dielectric elastomer actuator incorporating tunable bending stiffness
}

\author{
Lei Liu, ${ }^{1, *}$ Junshi Zhang, ${ }^{2, *, \dagger}$ Meng Luo, ${ }^{3}$ Bo Li $\odot,{ }^{4}+$ Chao Tang, ${ }^{5}$ Hualing Chen ${ }^{4}$ Zhichun Yang, ${ }^{2}$ Pengfei Li, ${ }^{1}$ and Dichen Li ${ }^{3}$ \\ ${ }^{1}$ School of Mechanical and Precision Instrument Engineering, Xi'an University of Technology, Xi'an 710048, China \\ ${ }^{2}$ School of Aeronautics, Northwestern Polytechnical University, Xi'an 710072, China \\ ${ }^{3}$ State Key Laboratory for Manufacturing Systems Engineering, Xi'an Jiaotong University, Xi'an 710054, China \\ ${ }^{4}$ Shaanxi Key Laboratory for Intelligent Robots, School of Mechanical Engineering, Xi'an Jiaotong University, Xi'an 710049, China \\ ${ }^{5}$ Institute for Future, Qingdao University, Qingdao 266071, China
}

(Received 19 December 2019; accepted 5 May 2020; published 20 May 2020)

\begin{abstract}
The superior ability of soft robotic systems is their good adaptability to complicated external environments without damaging themselves and their surroundings. Dielectric elastomers (DEs) are known as artificial muscles for soft actuations; however, rigid frames are commonly required to sustain the prestretch, highly limiting flexibility designs. Moreover, under high actuation voltage, DEs are susceptible to electrical breakdowns. Furthermore, pure-soft actuators cannot sustain deformations under external loads. Therefore, based on the fiber-reinforced DE films, we develop a cylindrical freestanding electro-pneumatic DE actuator (EPDEA), which combines the advantages of pneumatic and electric actuators. The finite element model (FEM) simulation is also performed to study the combined deformations (a combination of elongation and bending) of EPDEAs. Due to fiber reinforcement, we introduce the mechanical anisotropies in DE films, and realize the large directional bending deformations $\left(>180^{\circ}\right)$ and output forces under the electro-pneumatic actuation. Fiber-jamming suits (FJSs) are simultaneously developed for the EPDEA to obtain the variable stiffness capability. The EPDEA with the FJS unit can hold the end position under a weight of $200 \mathrm{~g}$ (which is almost 4 times the weight of EPDEA). The effects of structural parameters and supporting pressure on the actuation and stiffness tuning the performance of EPDEA are also investigated.
\end{abstract}

DOI: 10.1103/PhysRevResearch.2.023202

\section{INTRODUCTION}

Motors and pistons are often regarded as rigid driving components, which are frequently used to make traditional robotic systems. However, their adaptability is often sacrificed, which significantly lowers their capabilities and efficiency in unstructured and real-time variable scenarios. Inversely, soft robotic systems excel at adaption to the complicated and dynamic environments, due to their soft and compliant bodies, shock-absorbing characteristics, resilience, and morphological adaptability [1-6].

Flexible actuation technology is always one of the key issues in the soft robotic community, which has been developed as many potential applications, including cable-driven actuators [7,8], pneumatic and hydraulic actuators [9-12], shape memory alloy $[13,14]$, and dielectric elastomer actuators (DEAs) [15-19]. Among these, the cable drive actuator enjoys much attention in previous studies for its flexibility and accuracy, and is often used to power continuum robots

\footnotetext{
*These authors contributed equally to this work.

†junshi.zhang@nwpu.edu.cn

${ }^{\ddagger}$ liboxjtu@xjtu.edu.cn
}

Published by the American Physical Society under the terms of the Creative Commons Attribution 4.0 International license. Further distribution of this work must maintain attribution to the author(s) and the published article's title, journal citation, and DOI.
[20]. However, suffering from failures, a cable-driven multifreedom system requires many motors and complex routines for cables. Pneumatic actuators, like body muscles, are the most popular for their large strokes and high-output force. Besides, with the delicate design of flexible constraints, they are capable of realizing various actuations. Nevertheless, pneumatic actuators have a low response speed and control accuracy, especially when a fine adjustment of small motions is needed. Moreover, plenty of internal channels and external valves are required to achieve multifreedom deformations, which would make the systems more complicated and bulky.

Among the advantages of DEAs, there are impressive actuation strain, rapid actuation speed, high-compliance selfsensing, and low noise [21-24]. However, some of them are typically achieved at particularly extreme conditions, while other advantages require the detriment of other ones. Furthermore, although the DEAs can achieve significant deformation and output significant forces, they are generally accompanied with the unwelcome electric breakdown failure [25-27] and electromechanical instabilities [27-30], leading to low reliability for practical applications. In addition, DEAs are commonly attached to rigid and bulky frames to sustain the prestretch for large actuation strokes, which increases their rigidity and complexity. In summary, the technological readiness of DEA is still lower than that of other actuation principles mentioned before. In this research, we strive to overcome some of these drawbacks of DEA to ensure its wide application. 


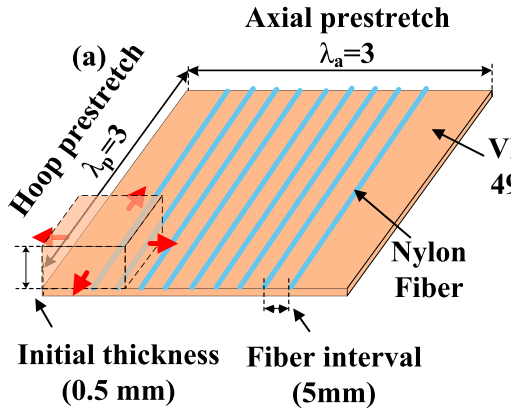

(d)

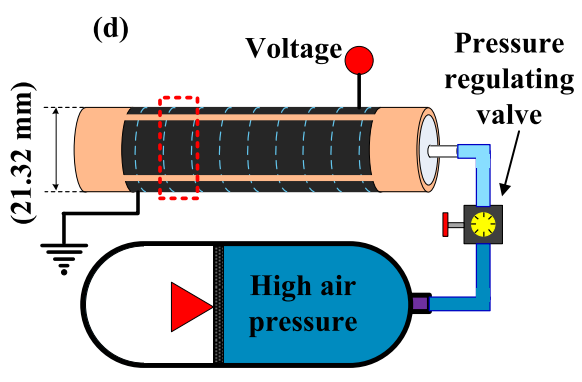

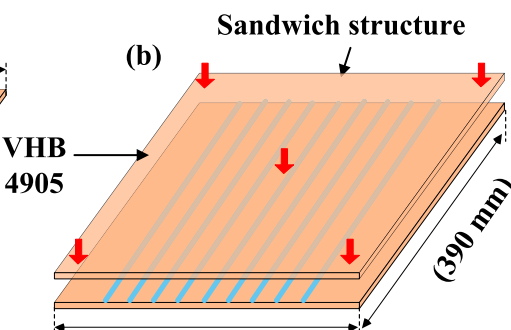

(100 mm)

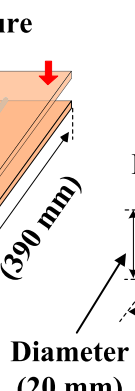

(20 mm)
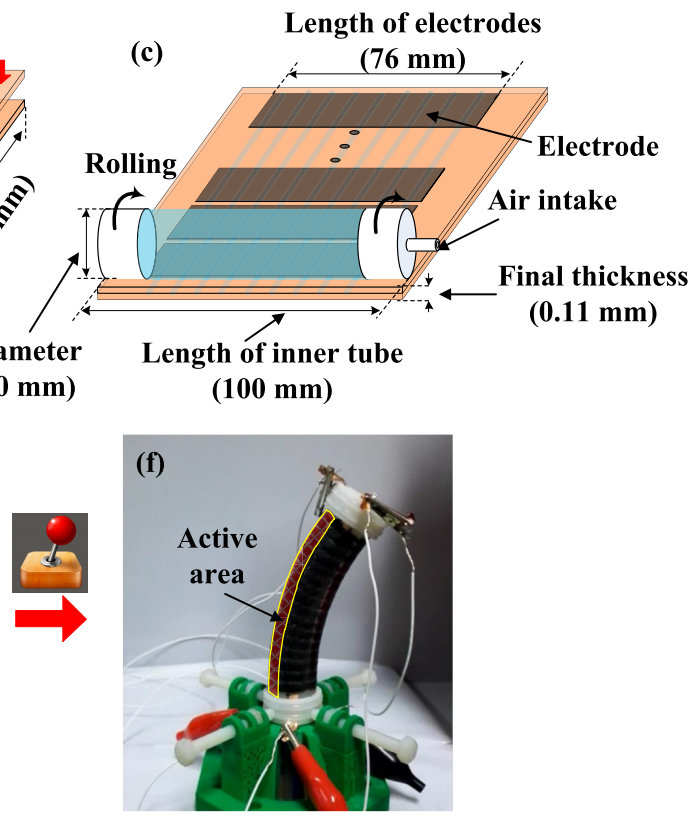

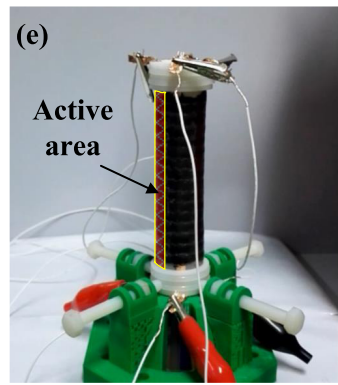

FIG. 1. The manufacturing process and actuation principle of the EPDEA. (a) Nylon fibers are parallelly placed on a stretched VHB 4905 with a prescribed distance to form a fiber layer. (b) The fiber layer is sandwiched by two VHB 4905 films with the same prestretch. (c) Both the surfaces of fiber-reinforced film are coated with compliant electrodes (carbon grease) and rolled into a cylinder through a pressure inner tube made of a VHB 4905 film, with a diameter of $20 \mathrm{~mm}$. (d) The EPDEA is inflated by air pressure to sustain the prestretch. (e) EPDEA reference state. (f) EPDEA actuated state.

During recent years, many DEAs combining pneumatic actuation with incompressible and compressible fluids have been fabricated [30-34]. Moreover, the cylinder bending and extending DEAs are simultaneously attempted to design [35-37]. Here, we present a kind of electro-pneumatic DEA (EPDEA), which features a large and fast actuation in both bending and elongation. The EPDEA is designed by drawing inspiration from the antagonistic mechanism of natural muscles, synergizing the advantages of both traditional DEAs and pure-pneumatic actuators. Compliant electrodes of DEAs are introduced to replace the internal pressure channels of purepneumatic actuators, forming a pure-soft actuator with a hollow shell structure. The EPDEA fully minimizes the bending stiffness and promises well-matched interactions with delicate and fragile objects. However, an actuator with a small stiffness is difficult to hold the deformation under the external perturbations. Different variable stiffness technologies (VSTs) for soft robotics were recently developed to overcome this problem, in which jamming is a common VST mechanism (which enables disordered, amorphous systems to switch reversibly between solidlike rigidity and fluidlike plasticity) for its fast response and large tunable range. Moreover, particle- and layer-jamming VSTs [38,39] have been adopted for various soft and continuum robotics, such as universal grippers [40], JamBot locomotion system [41], and minimally invasive surgical manipulators $[42,43]$. However, the layer- and particlejamming VSTs have relatively high initial stiffness or large weight and volume, which may be unsuitable to electroactive polymer systems, because they may fail to deform even in the unjamming state.

In this work, we develop a kind of fiber-jamming suit (FJS) for the EPDEA, which features low initial stiffness (in the unjamming state), light weight, and thin-shell structure.
The combined EPDEA-FJSs have several properties: (i) they incorporate electric and pneumatic actuation, which provides direct coupling of electric and pneumatic powers to achieve high-power and precise actuation with a low risk of electric breakdown failures. (ii) They have two actuation modes (bending and elongation). (iii) They fully simplify the inner structure as thin cylindrical shells and obtain large prestretch without relying on rigid frames. (iv) They are equipped with the FJS which enables a tunable stiffness.

\section{DESIGN AND PREPARATION OF THE EPDEA}

This section presents a design of an EPDEA based on fiberreinforced VHB 4905 films (see Fig. 1). VHB 4905 is a typical VHB-series DE material in the shape of a membrane, $0.5 \mathrm{~mm}$ thick. Under a high voltage across the thickness direction, the area of VHB 4905 expands while the thickness shrinks. As Figs. 1(a)-1(d) show, the fabrication of an EPDEA involves several steps. Inspired by pneumatic artificial muscles, this study adopts fiber reinforcements, such as by nylon fibers [44], to create mechanical anisotropies in VHB films that highly promote to achieve a natural musclelike unidirectional actuation. Nylon fibers with a diameter of $0.107 \mathrm{~mm}$ are evenly and parallelly distributed on a biaxially prestretched VHB film with a prescribed fiber interval [Fig. 1(a)]. A second DE film with the same prestretch is overlapped to make a sandwich structure (the nylon fibers lay between the two prestretched VHB films), as shown in Fig. 1(b). The compliant electrodes are then coated on the top side of the sandwich structure. The electrodes are patterned in rectangular areas separated by insulating gaps of $2-3-\mathrm{mm}$ width. The electrode areas stack on top of each other and radially align on two circumferential spans of the roll [45]. In order to obtain 


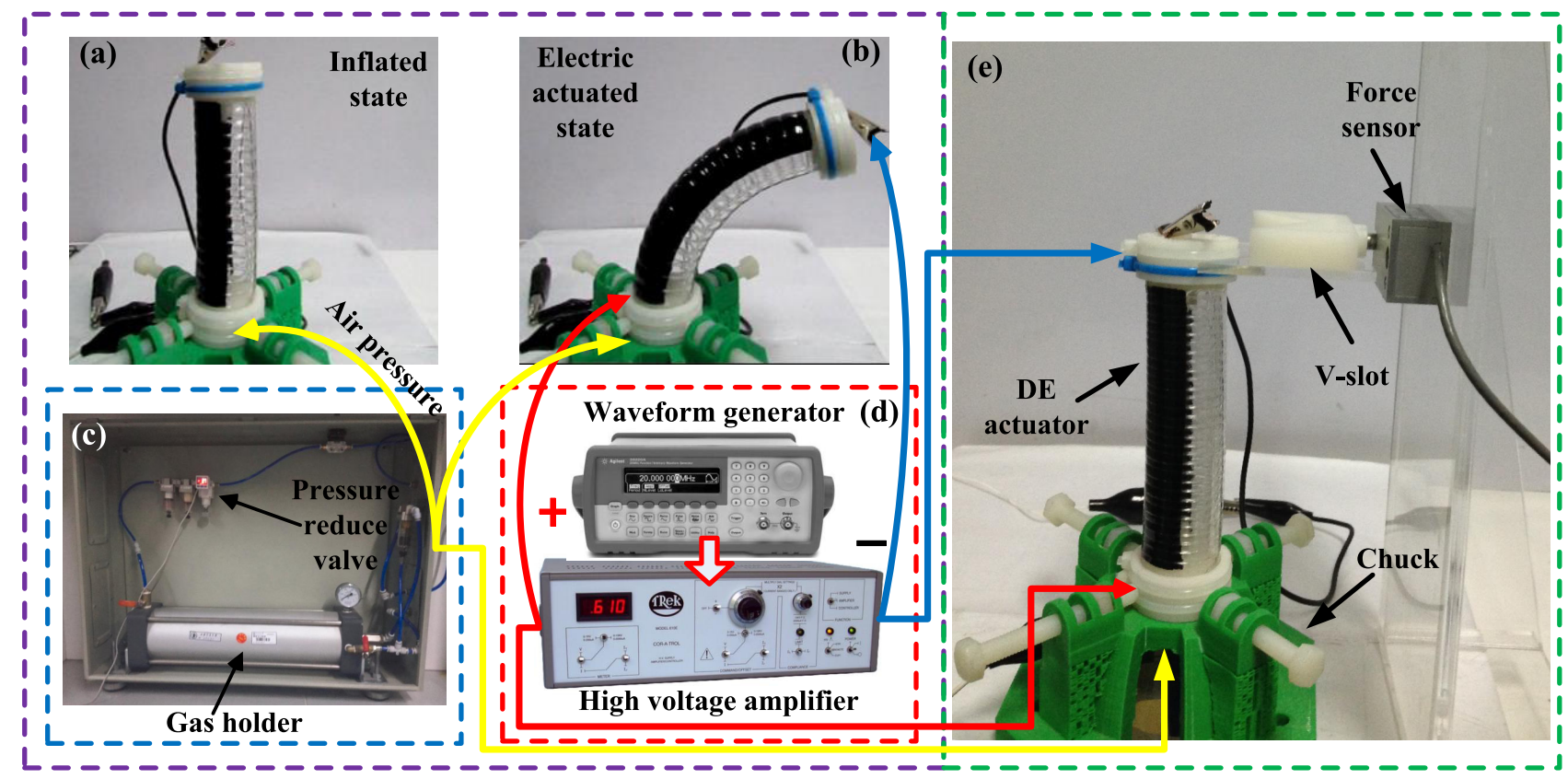

FIG. 2. The experimental setup. (a), (b), (e) Actuators fixed on a four-jaw chuck [fiber interval of $5 \mathrm{~mm}$ (left two) and $2.5 \mathrm{~mm}$ (right one), hoop prestretch of 3 , inner tube diameter of $20 \mathrm{~mm}$, electrode area of $180^{\circ}$, layer number of 6 , and supporting pressure of $35 \mathrm{kPa}$; (c) The air compressor pumps air into the gas holder to provide the EPDEAs with stable high pressure; (d) The voltage amplifier and the wave-form function generator that provides EPDEAs with electric power; (e) The GS0-500 force sensor for blocking force tests.

uniformly distributed electrodes, the serigraphy method was used to deposit carbon grease (MG Chemicals) by laying a hollowed-out mask onto the film. A single layer of VHB 4905 film is attached on pedestals [fabricated by three-dimensional (3D) printing, with a radius of $10 \mathrm{~mm}$ and a length of $12 \mathrm{~mm}$ ] to form a cylindrical pressure inner tube. The sandwiched DE is rolled along with a cylinder around the tube [Fig. 1(c)]. Finally, compressed air is pumped into the EPDEA through an air intake to sustain the prestretch [Fig. 1(d)]. Here we give a set of parameter reference values for manufacturing: The fiber interval is $5 \mathrm{~mm}$, the biaxial prestretch is $3 \times 3$ (where $\lambda_{p}$ is the hoop prestretch; $\lambda_{a}$ is the axial prestretch and prescribed as 3 in this research), the initial thickness of VHB 4905 is $0.5 \mathrm{~mm}$, the final thickness is $0.11 \mathrm{~mm}, 6$ layers of films are stacked in a roll, the length of electrode is $76 \mathrm{~mm}$, the number of electrodes is 24 (each circumferential span has 4 electrodes), the diameter of inner tube is $20 \mathrm{~mm}$, the diameter of the fabricated actuator is about $21.32 \mathrm{~mm}$, the initial length of the actuator equals that of the inner tube of $100 \mathrm{~mm}$, and the final length is not an independent parameter which relies on the input air pressure.

Considering the viscoelasticity of VHB films, the fabricated EPDEA is allowed to creep for $30 \mathrm{~min}$ before measurements. An EPDEA can be considered to have several repeated cylindrical units separated by the fibers [the red dotted box in Fig. 1(d)]. Also, a better actuation performance and electromechanical stability of DE has already been demonstrated in large prestretch states [27]. The large hoop prestretch is also an important factor in this design [45], which is investigated later in this paper. Air pressure is introduced to sustain the hoop prestretch to avoid severe buckling along the peripheral direction. Note that such an electro-pneumatic inhomogeneous deformation depends on several factors, such as prestretch, fiber intervals, distribution of electrodes, and diameters. Thus, according to these analyses on design criteria, effects of the prestretch, fiber intervals, distributions of electrodes, and diameters of the inner tube on the actuation performance of EPDEAs are considered. Section IV discusses in detail these effects.

The antagonistic mechanism of natural muscle groups (distributed in the hoop) is integrated into this design to realize an antagonistic bending actuation, as Figs. 1(e) and 1(f) show. Four electrodes are evenly distributed along the hoop of cylindrical actuator. When a high voltage is applied on a certain electrode (i.e., the active area), the EPDEA bends toward the opposite side of the active area. Also, owing to electro-pneumatic actuation, the EPDEA experiences a complicated volume variation, including an axial elongation and a continuum bending. By considering the complexity of the actuation mechanism, a FEM model of EPDEA is also introduced to further analyze and interpret the experimental results.

\section{EXPERIMENTAL SETUPS AND FEM SIMULATION METHODS FOR EPDEA}

Figure 2 shows the experimental setup of the system. It mainly includes a signal generator $\left(6811 \mathrm{~B}\right.$ Agilent $\left.^{\mathrm{TM}}\right)$ to produce various voltage signals, a voltage amplifier (model 610E TREK) to amplify the voltage signals, a gas holder connected to an air compressor that provides the EPDEAs with stable high pressure, and a pressure-reducing valve (SMC IR100001 ) with a precision of $0.1 \mathrm{kPa}$ mounted between the gas holder and the EPDEA to reduce the high pressure to the prescribed value. Moreover, a digital camera (Canon EOS 6D DSLR) records the experimental data. The bending angle is 
TABLE I. The parameters selected for experiments.

\begin{tabular}{lc}
\hline \hline Influence factors & Range of parameters \\
\hline Fiber interval $(\mathrm{mm})$ & $2.5,5,10,15$ \\
Hoop prestretch $\left(\lambda_{p}\right)$ & $2,3,4$ \\
Electrode area $(\mathrm{deg})$ & $90,135,180$ \\
Diameter of inner tube $(\mathrm{mm})$ & $15,17.5,20$ \\
Supporting pressure $(\mathrm{kPa})$ & $35,40,45,50,55$ \\
\hline \hline
\end{tabular}

obtained through IMAGEJ image analysis software, and a force sensor (GS0-500, Transducer Techniques) gains the blocking force data. For simplicity, the tests use each EPDEA with one active area, as shown in Figs. 2(a) and 2(b).

The experiments considered the effects of fiber interval, hoop prestretch, active electrode area, the diameter of the inner tube, and the supporting pressure. Table I lists the selected parameters, which can represent a wide range of information on electro-pneumatic actuation, in which the active electrode area is measured in terms of a central angle.

In order to further analyze and quantify the electropneumatic actuation of the EPDEA, the 3D finite-element models were established using the commercial finite-element software ABAQUS 6.14. A user subroutine (i.e., UMAT) [46] based on the ideal DE model was used to simulate the active region of the EPDEAs. The geometry of the EPDEA was built in the ABAQUS/CAE and was meshed linear hexahedron element with hybrid integration (ABAQUS element type C3D8H) for both active and inactive regions (Fig. 3). The geometry parameters were set following the experimental prototypes of the EPDEA. Note that, unless a specified parameter is investigated, the parameters of a reference group adopted in the experiments and simulation are as follows: fiber interval is $5 \mathrm{~mm}$, hoop prestretch is 3 , electrode area is $180^{\circ}$, the diameter is $20 \mathrm{~mm}$, layer number is 6 , and the supporting pressure is $35 \mathrm{kPa}$.

\section{RESULTS AND DISCUSSIONS FOR EPDEA}

The actuation performance of the EPDEA depends on the hybrid power. Furthermore, some key structural factors, such

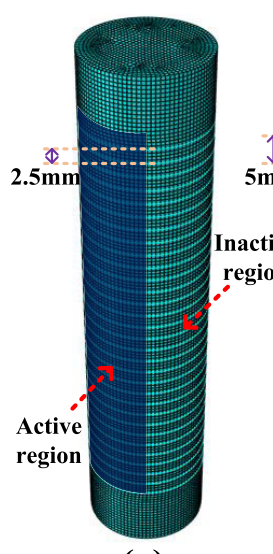

(a)

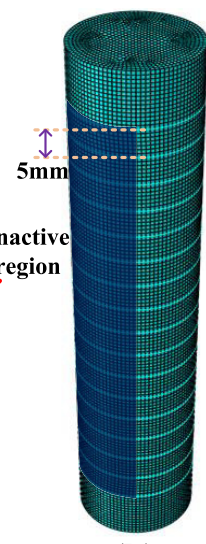

(b)

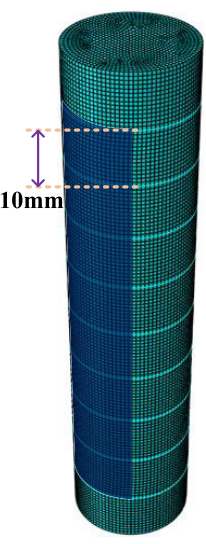

(c)

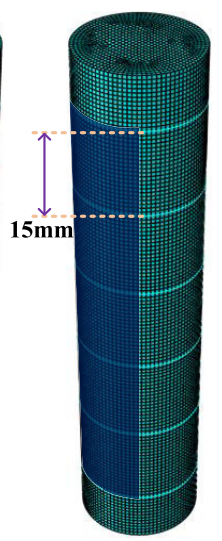

(d)
FIG. 3. The FEMs of the EPDEA: model (a), (b), (c), and (d) with fiber interval of $2.5,5,10$, and $15 \mathrm{~mm}$, respectively.
TABLE II. Influence of fiber interval on the elongation of the EPDEA.

\begin{tabular}{lrccc}
\hline \hline Fiber interval (mm) & \multicolumn{1}{c}{2.5} & 5 & 10 & 15 \\
\hline Length in simulation $(\mathrm{mm})$ & 100.3 & 104.7 & 110.6 & 114.4 \\
Length in experiment $(\mathrm{mm})$ & 98.3 & 100.3 & 106.6 & 108.7 \\
\hline \hline
\end{tabular}

as fiber intervals, hoop prestretch, active electrode area, and the diameter of the inner tube, affect the actuation performance. Therefore, this section divides the discussion into two parts. Part I deals with the effects of structural factors on the actuation performance under a certain actuation condition. Part II explores the characteristics of the electro-pneumatic actuation, setting the structural parameters as constants.

As is known, DEs are homogeneous and isotropic materials. However, subjected to air pressure, DEs experience a nondirectional and balloonlike inflation that reduces their actuation efficiency. On the other hand, with fiber reinforcements [Figs. 4(a)-4(d)], the material anisotropy is obtained, which contributes to achieving a unidirectional deformation like natural muscles. In Figs. 4(e)-4(h), the simulations are consistent with the experimental results concerning the fiber density effect. In Figs. 4(e)-4(1), both the simulation and experimental results demonstrate that a large fiber interval results in obviously drumlike inflation in the hoop direction, magnifying the geometric nonlinearity.

Moreover, under the same pressure, such hoop inflation is helpful to gain a significant elongation (see Table II). In other words, a high fiber density increases the stiffness in both hoop inflation and axial elongation, as Figs. 4(e)-4(h) show. According to Figs. 4(i)-4(1), under a constant electropneumatic actuation, the low fiber density would lead to a large bending angle. From the perspective of the mechanics of materials, for large fiber interval, the actuator is supposed to have a great bending stiffness due to its large diameter. However, the significant hoop inflation contributes to their prestretch in hoop direction, which nonuniformly increases the electric field applied to DEs under the same voltage.

The low fiber density would lead to a high risk of breakdown failures [Figs. 4(o) and 4(p)] because of the introduced loop inflation. By decreasing the fiber intervals from 15 to $2.5 \mathrm{~mm}$, the breakdown voltage rises from 5220 to $7690 \mathrm{~V}$. The EPDEA with a fiber interval of $5 \mathrm{~mm}$ obtains the maximum bending angle [over $180^{\circ}$, Fig. 4(n)]. It is mainly because the high fiber density (fiber interval of $2.5 \mathrm{~mm}$ ) may severely enhance the stiffness of EPDEAs, while the low fiber density (fiber interval of 10 and $15 \mathrm{~mm}$, respectively) may lead to electric breakdowns with the voltage increases. Moreover, the FEM models are simultaneously used to predict the breakdown position [Fig. 4(o)]. DE breakdown failures feature severely electromechanical coupling. Thus, the failure point is the most likely to overlap with the maximum strain point. With consideration of the gravity of EPDEA, the maximum strain in simulation occurs in the root segment of the actuator, which is the breakdown area observed in experiments [Fig. 4(p)].

Figures 5(a) and 5(b) show a systematic investigation on the maximum actuation performance; that is, both the 


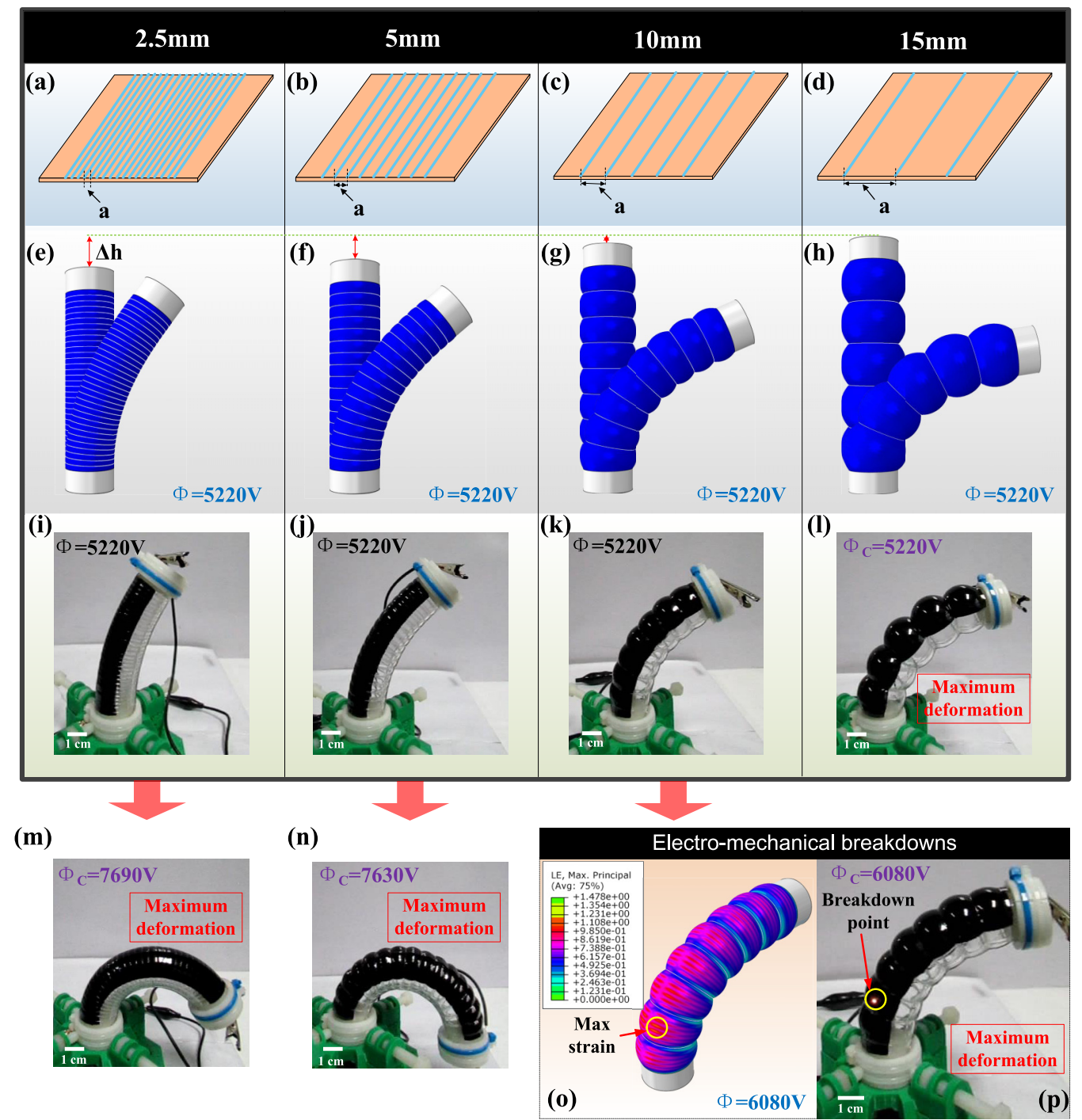

FIG. 4. Effect of fiber density on electro-pneumatic actuation (constant pressure of $35 \mathrm{kPa}$, hoop prestretch of 3, the diameter of the inner tube of $20 \mathrm{~mm}$, layer number of 6 , and electrode area of $180^{\circ}$ ): (a)-(d) different fiber density adopted to investigate; (e)-(l) simulation and experimental results on bending actuation with the different fiber density under $5220 \mathrm{~V}$; $(\mathrm{m})-(\mathrm{p})$ maximum actuation before their electromechanical breakdown failure occurs ( $\Phi C$ is critical breakdown voltage).

maximum bending angle and blocking force increase initially and subsequently decrease as the fiber density increases. Although the fiber interval influences the breakdown voltage, the breakdown field strength is not sensitive to this design parameter. Fiber reinforcements and supporting pressure enhance the maximum bending deformation and blocking force of the EPDEA and allow the actuator to have large hoop prestretch without introducing any rigid components, so that the performance of EPDEA is not affected. According to Figs. 5(c) and 5(d), the bending angle and blocking force increase by seven times and nine times, respectively, as the hoop prestretch increases from 2 to 4 due to a considerable Maxwell stress produced by a high level of prestretch. The reasons are as follows. A high level of prestretch leads to the small value of the thickness of VHB 4905. Under the same high voltage, a small thickness will generate a significant Maxwell's stress. Note that the increase in prestretch is demonstrated to be gained from the prescribed hoop direction and the axial direction.
As Table III summarizes, under the same supporting pressure of $35 \mathrm{kPa}$, a high level of hoop prestretch leads to a large elongation in the axial direction, mainly because of the incompressibility of DEs. With the same layer number, a large hoop prestretch would result in a small cross-section area of the EPDEA (cylindrical shell), which leads to high axial stress under the same air pressure. In other words, prestretching the VHB film in the hoop direction can reduce the axial stiffness.

Compared with typical pneumatic actuators, compliant electrodes are introduced to replace the internal pressure chambers in the EPDEA. From the perspective of manufacturing, the complexities lie in the arrangement of electrodes and chambers for these two kinds of actuators, respectively. In our design of EPDEA, the position and width of the electrodes are obtained from calculations. The width of the electrode area is deemed as incrementally increased, leading to a radial alignment on two circumferential spans of the roll. Moreover, as Fig. 6(a) shows, the distributions of compliant electrodes can fully correlate with the distributions of 

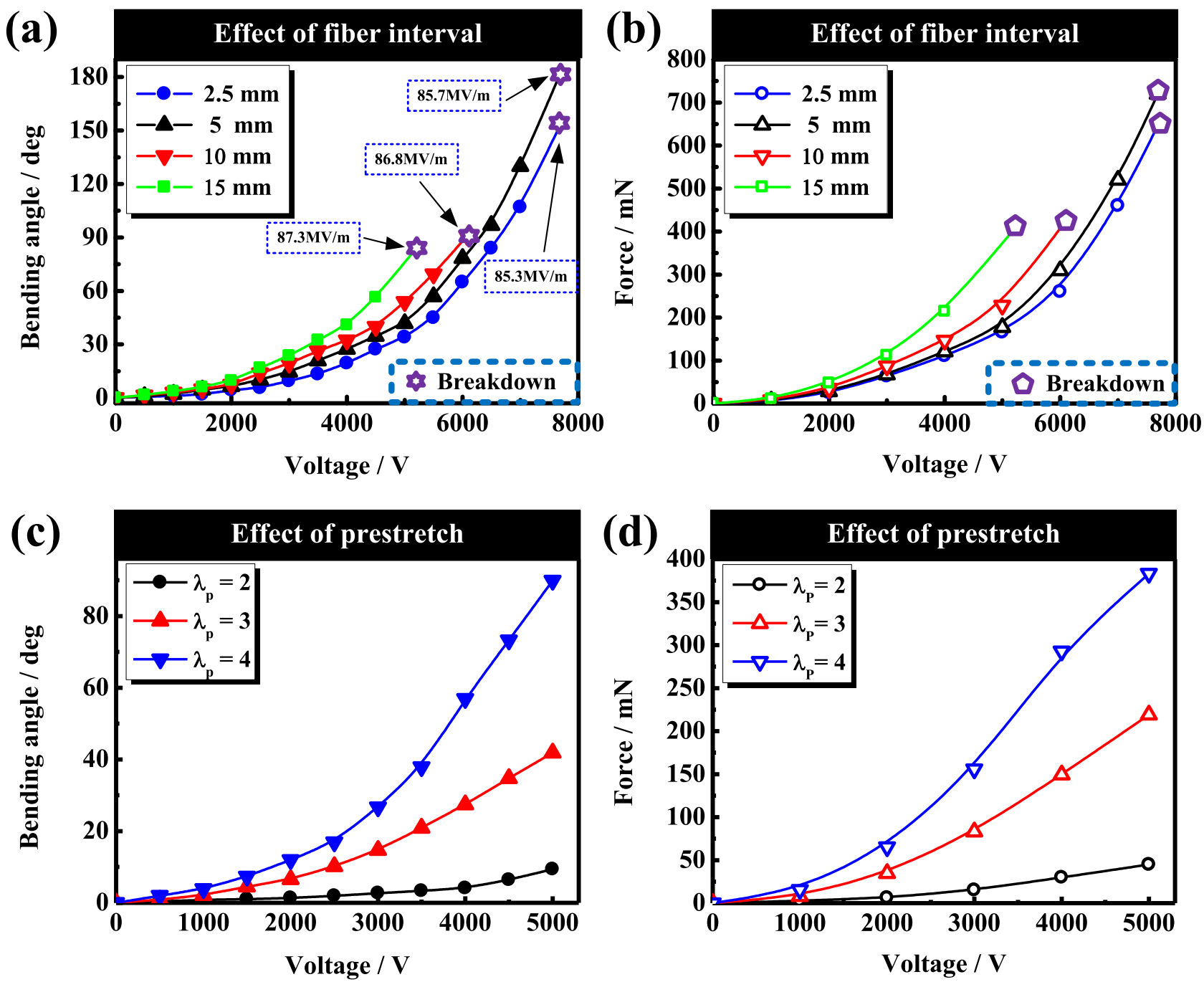

FIG. 5. (a), (b) The measured bending angles and blocking forces of EPDEA varying the voltage; (c), (d) Effect of hoop prestretch on the bending angles and blocking forces of the EPDEA varying the voltage (constant pressure of $35 \mathrm{kPa}$, fiber interval of $5 \mathrm{~mm}$, inner tube diameter of $20 \mathrm{~mm}$, layer number of 6 , electrode area of $180^{\circ}$ ).

chambers in pneumatic actuators. According to the antagonistic bending mechanism, the number of active electrodes must be considered for exploring the actuation performance of the EPDEA. Here, three typical and different active areas (central angle of $90^{\circ}, 120^{\circ}$, and $180^{\circ}$, which correlate with 4,3 , and 2 chambers in pneumatic actuators, respectively) are adopted to quantify such an effect on the actuation performances of EPDEA. As Figs. 6(b) and 6(c) shows, the bending angle and block force rise by 50 and $30 \%$, respectively, with the increase of active region from $90^{\circ}$ to $180^{\circ}$. Note that the distribution of different chambers in typical pneumatic actuators would cause the variation of bending or axial stiffness, resulting from the different inner structures. However, the mechanical properties of EPDEAs, such as bending or axial stiffness, are almost independent of the distribution of electrodes, due to the negligible shear modulus of compliant electrodes.

The soft bending actuators with multi-degrees of freedom are often expected for medical applications, such as minimally invasive surgical robotic systems. Miniaturization of the diameter $d$ is required, and it can be easily realized during the manufacturing process because it is not limited by the mold size and never troubled by gas bubbles and insufficient demolding strength, as Fig. 7(a) shows. At a constant pressure, the pneumatic power highly depends on the variation of volume. Thus, the effect of miniaturization on the coupled actuation is investigated under the constant pressure of $50 \mathrm{kPa}$. Figs. 7(b) and 7(c) show that, in a range of diameters from 15 to $20 \mathrm{~mm}$, larger diameter ensures superior performance in blocking force, and results in a large bending angle as well. Figure 7(a) schematically illustrates that under a constant layer number, a small diameter $d$ of EPDEA naturally requires less DE films and leads to a small transverse area of the DE roll, which could result in significant stress under the same axial supporting force induced by pressure. A large

TABLE III. Effect of the hoop prestretch on the elongation of the EPDEA.

\begin{tabular}{lccc}
\hline \hline Hoop prestretch & 2 & 3 & 4 \\
\hline Length $(\mathrm{mm})$ & 79.6 & 100.3 & 117.6 \\
\hline \hline
\end{tabular}




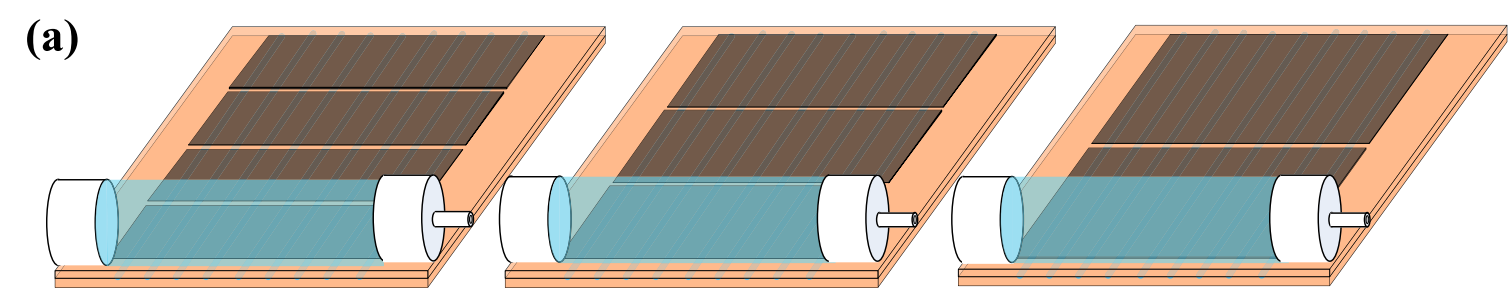

Four

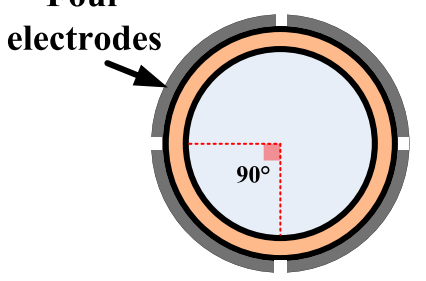

Three

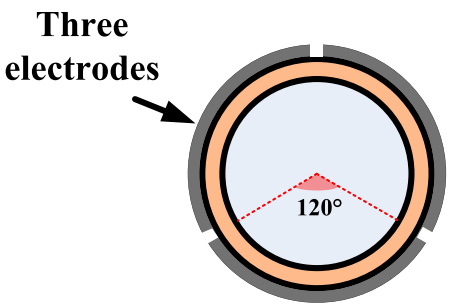

Two

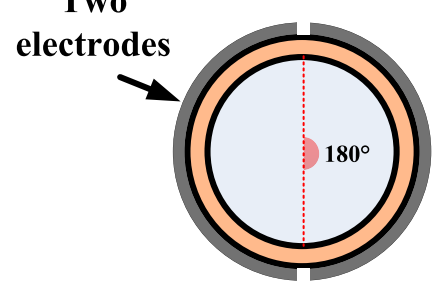

(b)

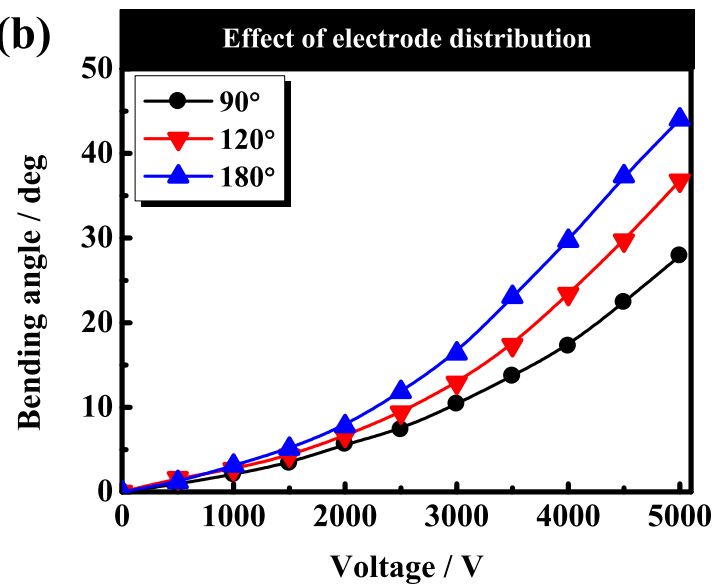

(c)

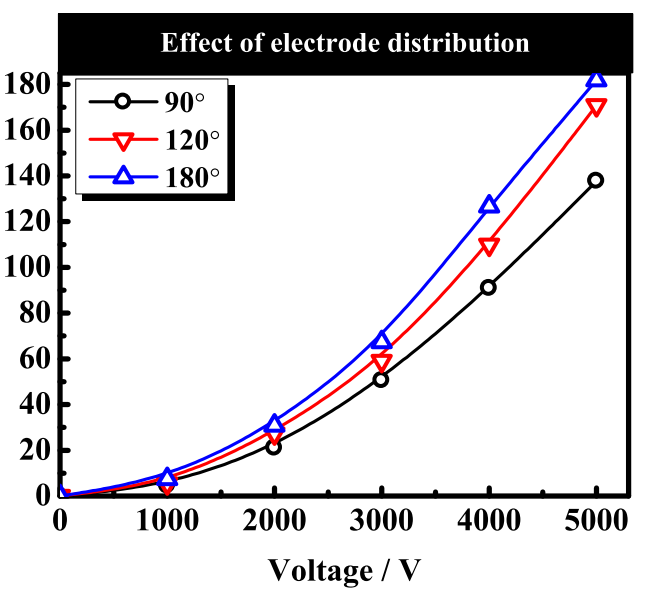

FIG. 6. (a) The different electrode distributions in design and manufacturing; (b), (c) Effect of electrode distribution on the bending angle and blocking force, respectively (constant pressure of $35 \mathrm{kPa}$, fiber interval of $5 \mathrm{~mm}$, hoop prestretch of 3 , inner tube diameter of $20 \mathrm{~mm}$, layer number of 6).

diameter, on the other hand, provides a large pressure area, which improves the supporting force along the axial direction. From these two opposite views, the diameter impacts the axial stress, which solely determines the elongation under a given pressure.

According to Table IV, larger diameter leads to a large axial elongation, which further results in a high Maxwell stress under the same voltage. Therefore, to a specific range of diameters from 15 to $20 \mathrm{~mm}$, a large diameter is susceptible to gain a large bending angle, despite its high bending stiffness. In general, under constant pressure, almost all the parameters, such as the fiber distribution, hoop prestretch, and diameter can affect the axial stiffness (see Tables II-IV). Due to the complicated electromechanical coupling, a low axial stiffness leads to a superior actuation performance with a given electropneumatic load.

In the above analyses, the pneumatic pressure is set as a constant for exploring the actuation performance of EPDEA. Thus, this actuation mode can be defined as an electric control mode. Under the fixed pressure of $35 \mathrm{kPa}$, the simulation agrees well with the experimental results when the applied voltage varies from 4500 to $6000 \mathrm{~V}$ [Fig. 8(a)]. In Fig. 8(b), the bending angle and axial elongation determine the end position of an EPDEA. However, in the electric control mode, the deformation of the EPDEA can almost be regarded as pure bending. When the voltage rises from 4500 to $6000 \mathrm{~V}$, the bending angle increases by $139 \%$ (from $33^{\circ}$ to $79^{\circ}$ ); however, the axial elongation grows by only $8 \%$ (from 72 to $78 \mathrm{~mm}$ ). In Figs. 8(c) and 8(d), under a constant voltage, the pneumatic pressure is considered as a tunable actuation to reveal more features of the pressure control mode. By varying the pressure from 35 to $50 \mathrm{kPa}$, the results of FEM simulations are further verified in a broad pressure range by the experimental results at the constant voltage of $5000 \mathrm{~V}$. Under increasing pneumatic pressure, the deformation of the EPDEA has remarkable axial elongation and bending. When the pressure varies from 35 to $50 \mathrm{kPa}$, the bending angle increases by $91 \%$ (from $41^{\circ}$ to $79^{\circ}$ ), and the axial elongation rises by $40 \%$ (from 72 to $102 \mathrm{~mm}$ ). This actuation mode features an obviously combined deformation like the capturing behavior of the "frog tongue." Therefore, the actuation of EPDEA essentially includes three modes: mode (i) is dominated by electric actuation, where the pressure is set as a constant. In this mode, the deformation mainly manifests an almost pure bending; mode (ii) is primarily controlled by pneumatic actuation under a given voltage, and the deformation shows a sort of combination of both bending and elongation. Besides, another mode of pure elongation can also be realized by applying the high voltage 
(a)
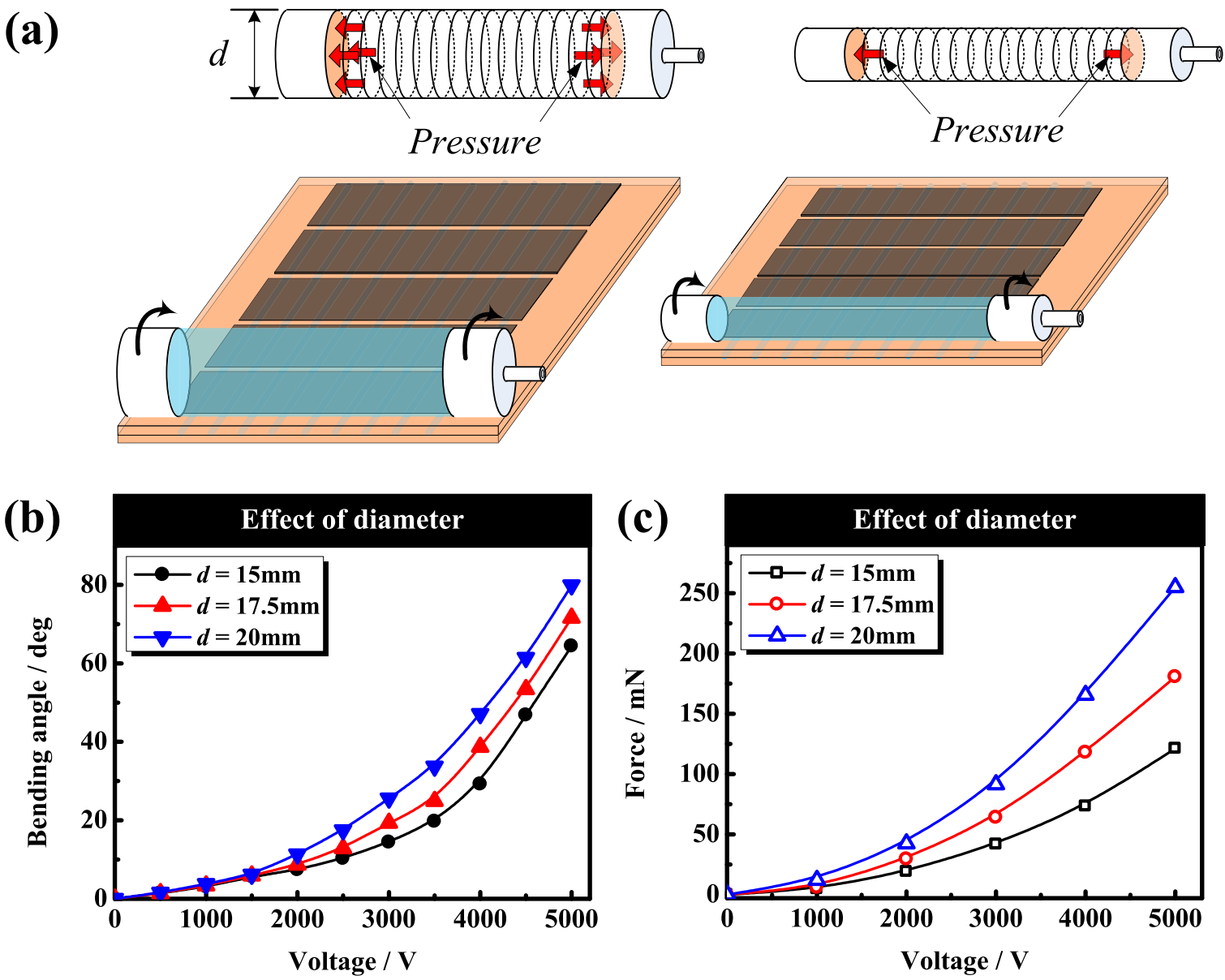

FIG. 7. Effect of the diameter of inner tube on the actuation of EPDEA: (a) The difference of manufacturing process and supporting forces between actuators with various diameters d; (b), (c) Effects of the diameter on the bending angle and blocking force varying the voltage (constant pressure of $35 \mathrm{kPa}$, fiber interval of $5 \mathrm{~mm}$, hoop prestretch of 3 , electrode area of $180^{\circ}$, layer number of 6).

to all the electrodes in the EPDEA or solely applying the pressure.

As previously mentioned, the actuation of EPDEA includes two modes. Such a two-mode actuation would highly enlarge the workspace of the end effector of EPDEA, which is reflected in Fig, 9(a). In the following, we further explore the effect of pneumatic pressure on the blocking force of EPDEA. As Fig. 9(b) shows, the high pressure remarkably improves the blocking force under a constant applied voltage. Thus, the electro-pneumatic coupling actuation method is demonstrated to be able to improve the insufficiency of the output force of conventional DEAs. By comparing two actuation modes, some conclusions can be obtained. The electric control mode has a fast response and high control resolution. Thus, this mode is probably required for the fine-tuning of actuation. On the other hand, the pneumatic control mode excels at high power output; therefore, it can be considered as a sort of

TABLE IV. Effect of the diameter of inner tube on elongation of the EPDEA.

\begin{tabular}{lccc}
\hline \hline Diameter of inner tube $(\mathrm{mm})$ & 15 & 17.5 & 20 \\
\hline Length $(\mathrm{mm})$ & 112.3 & 123.3 & 136.7 \\
\hline \hline
\end{tabular}

coarse tuning of actuation. Also, pneumatic control mode can lower the demand for high voltages, and reduce the risk of electrical breakdowns of DE membranes.

\section{DESIGN OF FJSs}

The important feature of the EPDEA is the soft and shell-like hollow structure, which significantly reduces the structural stiffness and provides well-matched and safe interactions between the actuator and environment. However, the lower stiffness of soft actuators results in an inability to hold their actuations and positions after the external actuation is removed. Therefore, we develop a variable stiffness unit for the EPDEA based on fiber-jamming mechanism, i.e., the fiber-jamming suit (FJS), which uses frictions (between fiber layers, and between fibers and membranes) by generating a vacuum. The jamming pressure $P_{\text {jam }}$ is defined as the negative pressure difference between the actuator core chamber and the ambient [the red region in Fig. 10(a,iii)] and represents the vacuum degree in this region. The FJS mainly consists of several fiber-jamming rings attached in series along the axial direction with a prescribed length of the overlap [Fig. 10(a)]. The fiber-jamming ring is made of plenty of nylon fibers fixed along the hoop of an adhesive tape. The patterned fiberjamming rings are sandwiched in a sealed vacuum region (red 


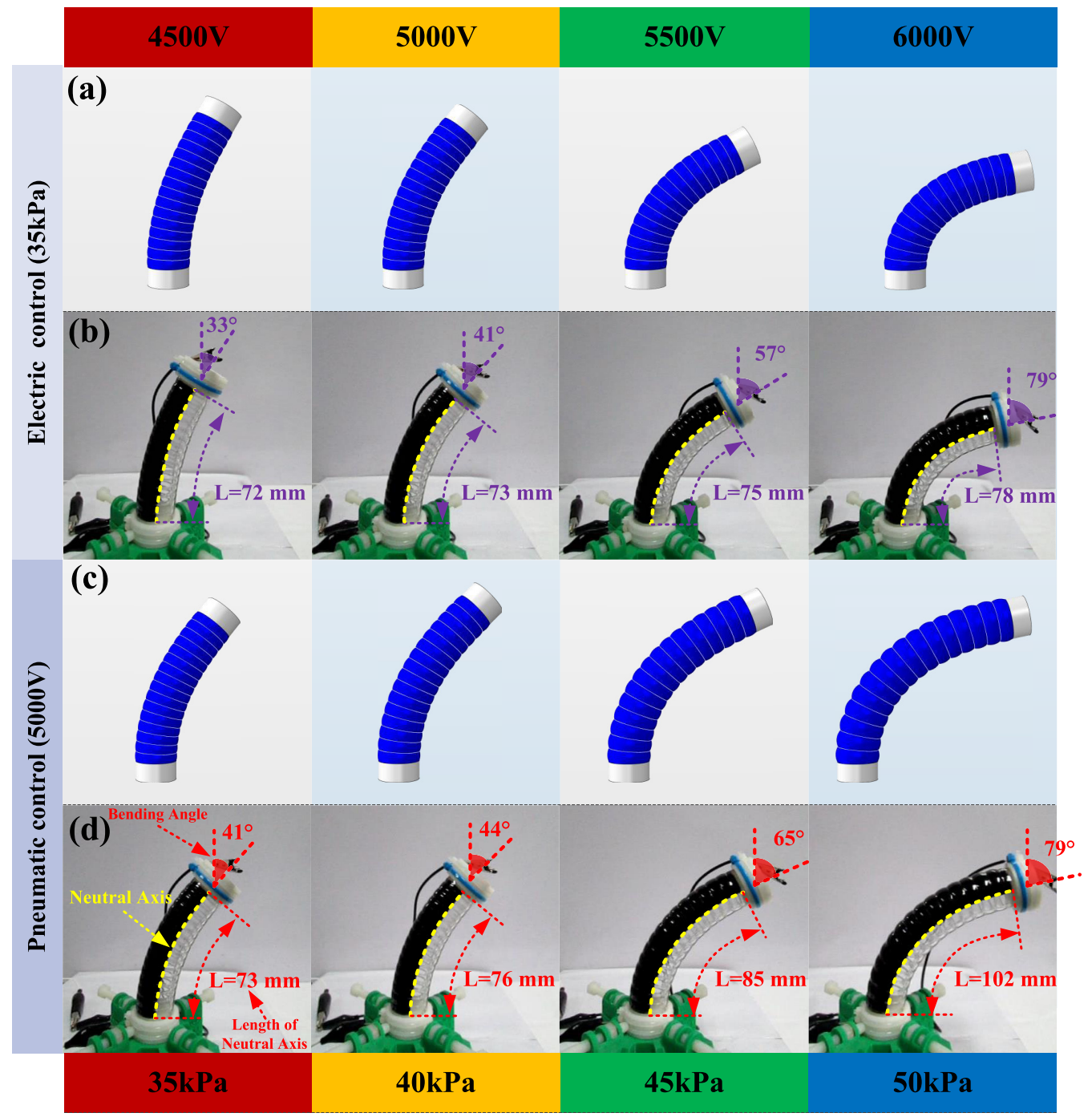

FIG. 8. The simulation and experimental results on the two-mode controlled actuation: (a), (b) electric control, constant pressure of $35 \mathrm{kPa}$; (c), (d) pneumatic control, constant voltage of $5000 \mathrm{~V}$ (sample manufacturing parameters: fiber interval of $5 \mathrm{~mm}$, hoop prestretch of 3 , inner tube diameter of $20 \mathrm{~mm}$, electrode area of $180^{\circ}$, layer number of 6 ).

(a)

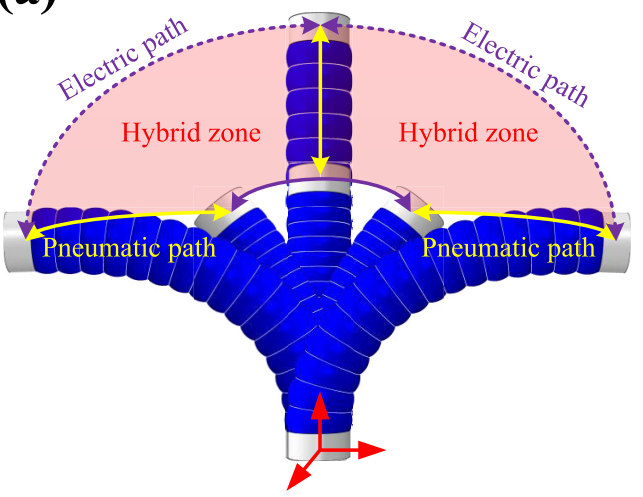

(b)

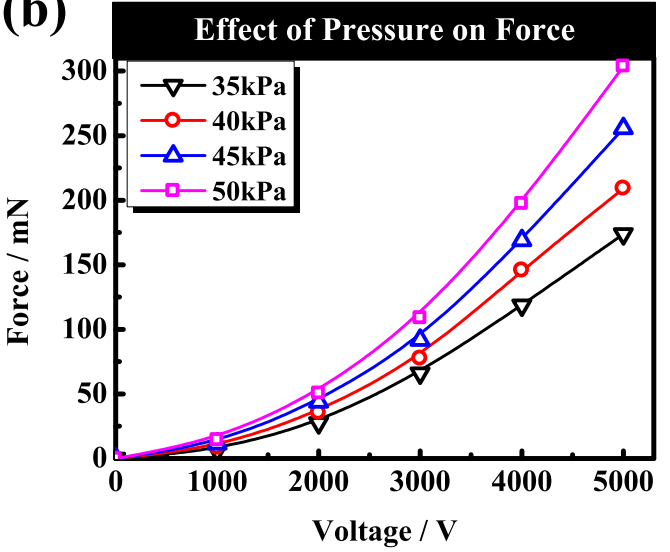

FIG. 9. Effect of pneumatic pressure on the hybrid actuation: (a) The enlarged workspace and the deformation features of two actuation modes (voltage 0-5000 V, pressure 35-55 kPa); (b) The effect of pneumatic pressure on the blocking force (sample parameters: fiber interval of $5 \mathrm{~mm}$, hoop prestretch of 3 , inner tube diameter of $20 \mathrm{~mm}$, electrode area of $180^{\circ}$, layer number of 6 ). 


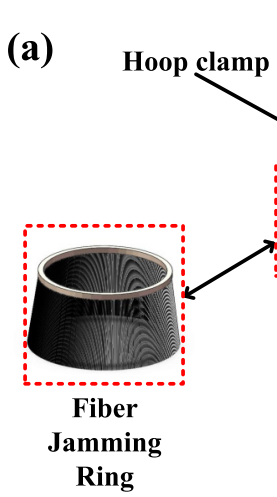

(b)

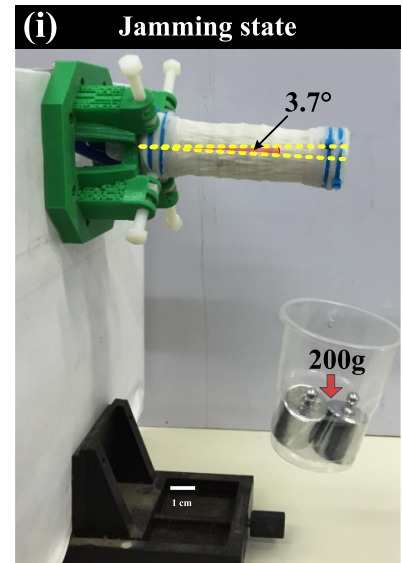

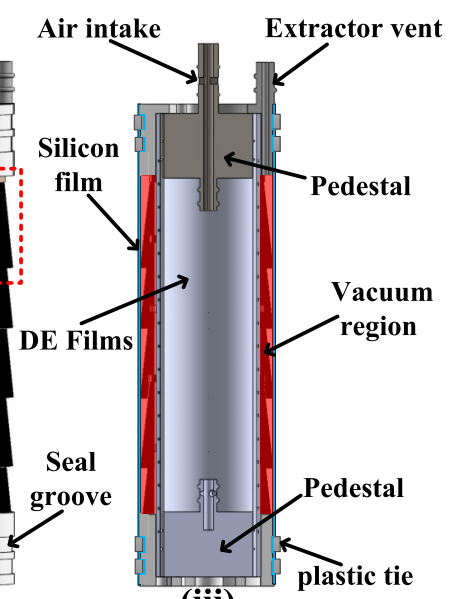

(iii) (i)

(ii) (ii) Unjamming state

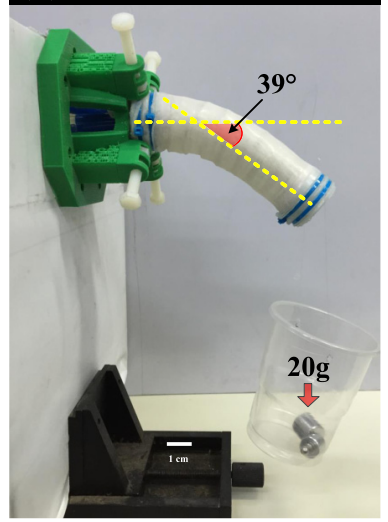

FIG. 10. (a) (i-iii) The 3D design of FJS for the EPDEA; (b) (i-ii) The variable stiffness performance introduced by the FJS: comparison of bending angles of EPDEA in the jamming and unjamming state under external loads.

region), which is jointly established by a silicone film (Ecoflex 0020, $1 \mathrm{~mm}$ thick) and the outer side of the EPDEA.

Compared with the particle-jamming mechanism, due to the significant difference between the tension and compression processes of fibers, the fiber jamming has obvious anisotropy. As a result, fiber jamming is more suitable to establish flexible and directional constraints in a soft system. Thus, the constraint highly depends on the conditions of deformation, which should be firstly determined during the design of FJS. Under electro-pneumatic actuation, the EPDEA would present bending, elongation, or their combination. However, the length of cylindrical generatrices would change when the EPDEA experiences the bending or the elongation. Therefore, the FJS is designed to constrict the length of cylindrical generatrices flexibly. When applying the vacuum, the friction introduces flexible constraints along the generatrix of the EPDEA. Thus, the overall stiffness can be continuously modulated by tuning the vacuum level.

As Fig. 10(b) shows, in the unjamming state, the EPDEA cannot even sustain the small load of $20 \mathrm{~g}$, presenting a bending angle of $39^{\circ}$. However, in the jamming state $\left(P_{\text {jam }}=\right.$ $96.9 \mathrm{kPa})$, the EPDEA is stiffened to show a small bending angle of $3.7^{\circ}$ under the large load of $200 \mathrm{~g}$ [Fig. 10(a)].

\section{EXPERIMENTAL STUDY AND DISCUSSIONS OF THE FJS}

An ideal jamming suit for a soft system (especially with a lower force output) is expected to be highly stiffened in the jamming state. On the other hand, it should have low impedance in the unjamming state to make sure of large deformations of soft actuators. Therefore, in this section, we evaluate the performance of the FJS when the EPDEA is in the unjamming state. As presented in Figs. 11(a) and 11(b), under a given driving condition (voltage: $6000 \mathrm{~V}$, air pressure: $35 \mathrm{kPa}$ ), the FJS would reduce the bending angle to a certain extent. It is stated that the FJS introduces bending resistances that mainly consist of the following aspects: (i) the friction between fibers; (ii) the friction between fibers and DE membranes; (iii) the elastic resistance of the sealing membrane; (iv) the bending stiffness of fibers. Figure 11(c) quantifies the effect of jamming suit on the bending angle for the unjamming state; according to the results, the loss of bending angle is demonstrated to be within $20 \%$.

On the other hand, the performance of the variable stiffness of EPDEA is investigated by varying fiber diameters and $P_{\text {jam. }}$. In Fig. 12(a), we introduce a three-point bending test to evaluate the performance of the FJS. A massive displacement represents a low stiffness under a given situation. As Fig. 12(b) shows, under the same $P_{\text {jam }}$, a large fiber diameter can lead to lower stiffness mainly because a small fiber diameter results in a broad frictional contact area between fibers, which increases the friction between fiber rings and strengthens the flexible constraints. Moreover, the stiffness can be continuously tunable through varying $P_{\text {jam }}$. As shown in Fig. 12(b), the stiffening behavior is highly nonlinear, where a dramatic loss of stiffness occurs when $P_{\text {jam }}$ is lower than $20 \mathrm{kPa}$. In general, in practical applications of FJSs, the fibers with a small diameter are suggested to obtain a better jamming performance. Moreover, the approximately linear and continuous stiffness variation can be achieved when $P_{\text {jam }}$ is higher than $20 \mathrm{kPa}$.

\section{CONCLUSIONS}

In this study, we develop an electro-pneumatic dielectric elastomer actuator (EPDEA) based on fiber-reinforced DE films. The fiber-jamming suit (FJS) is also designed for the EPDEA to obtain a variable stiffness capacity. Inspired by the antagonistic mechanism of natural muscles, the EPDEA is fabricated to realize its multidirectional bending and elongation actuation. The electro-pneumatic actuation merits two actuation modes: (i) the voltage-dominated mode has high control accuracy and fast response; (ii) the pressure-dominated mode excels at the high output force. Moreover, the dualmode actuation would also highly enlarge the workspace of the end effector of EPDEA. Also, experiments and FEM simulation are carried out to investigate the influencing factors on the actuation performances. The results are as follows. (a) A high fiber density results in a large maximum actuation before breakdown failures; (b) when the hoop prestretch increases from 2 to 4, the bending angle and blocking force highly rise by sevenfold and ninefold, respectively; (c) with the increase of electric active area from $90^{\circ}$ to $180^{\circ}$, the bending angle 
(a)

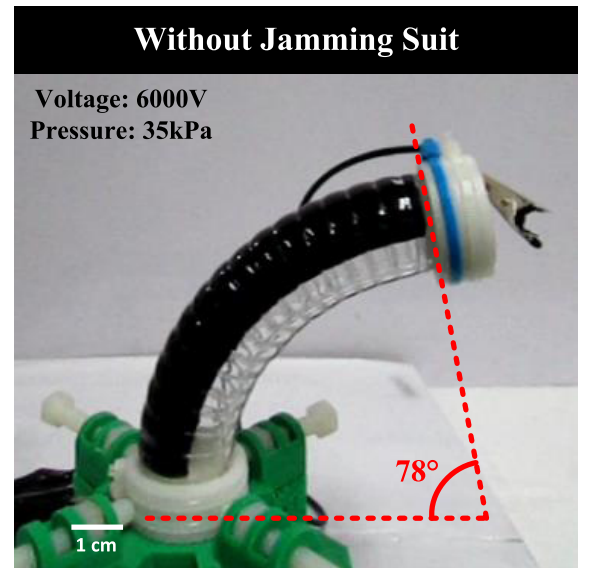

(b)

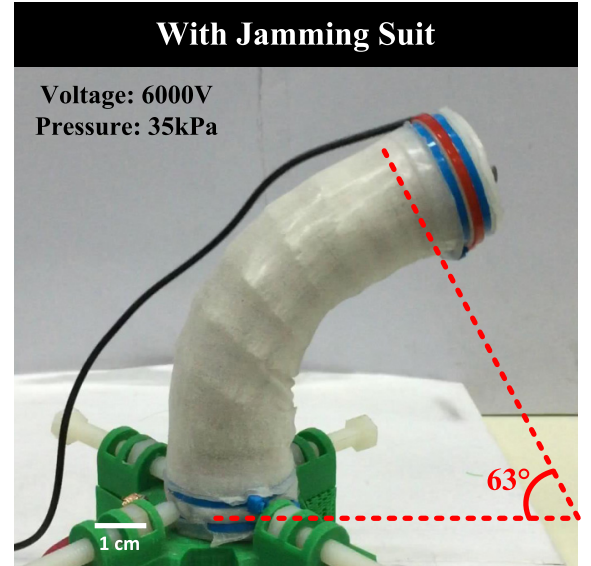

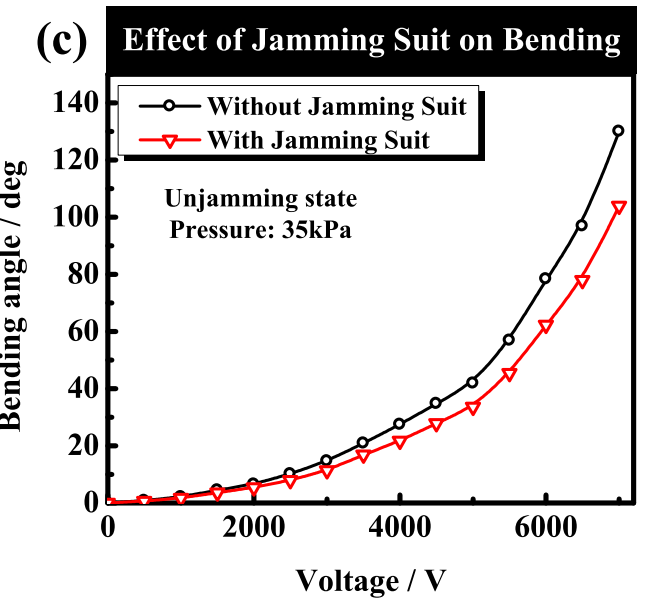

FIG. 11. The effect of FJSs on the actuation in the unjamming state. (a): Bending angle without jamming suit under prescribed driving condition (voltage: $6000 \mathrm{~V}$, air pressure: $35 \mathrm{kPa}$ ); (b): Bending angle owing to the resistance introduced by the FJS; (c): Effect of FJSs on the bending angle varying the voltage (EPDEA manufacturing parameters: fiber interval of $5 \mathrm{~mm}$, hoop prestretch of 3, inner tube diameter of $20 \mathrm{~mm}$, electrode area of $180^{\circ}$, layer number of 6$)$.

and blocking force increase by 50 and 30\%, respectively; (d) within a specific range of diameter, a large diameter induces a large bending angle, despite its bending stiffness; (e) the large air pressure enhances the actuation performances remarkably, especially the blocking force. Finally, a sort of FJS is developed for the EPDEA to tune the stiffness, which features a low initial stiffness (in the unjamming state), light weight, and thin shell-like structure. The nonlinear relationship between
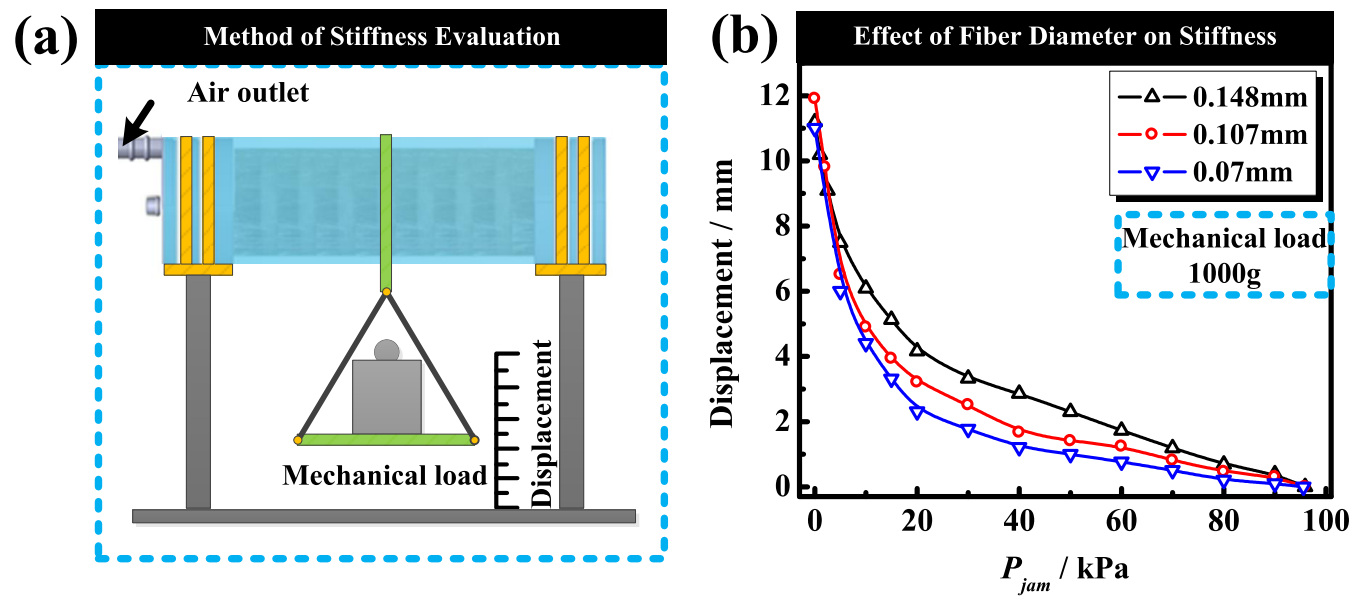

FIG. 12. Investigation on variable stiffness performance and the related affecting factors: (a) The stiffness evaluation method; (b) The influence of fiber diameters and $P_{\text {jam }}$ on the performance of variable stiffness (EPDEA manufacturing parameters: fiber interval of $5 \mathrm{~mm}$, hoop prestretch of 3 , inner tube diameter of $20 \mathrm{~mm}$, electrode area of $180^{\circ}$, layer number of 6 ). 
the stiffness and $P_{\text {jam }}$ is investigated, which may be affected by the positive feedback of friction. The research results can be applied to guide the design and fabrication of soft actuators with variable stiffness, and advance the development of the soft robotics community. Future works may include an extensive study on the quantitative frictional contact model for the fiber-jamming suit.

\section{ACKNOWLEDGMENTS}

This research was supported by the National Natural Science Foundation of China (Grants No. 51805413 and No. 11802222), the Fundamental Research Funds for the Central Universities (Grant No. G2019KY05104), and the 111 Project (Grant No. BP0719007).
[1] E. W. Hawkes, L. H. Blumenschein, J. D. Greer, and A. M. Okamura, Sci. Robot. 2, eaan3028 (2017).

[2] M. Cianchetti, C. Laschi, A. Menciassi, and P. Dario, Nat. Rev. Mater. 3, 143 (2018).

[3] R. F. Shepherd, F. Ilievski, W. Choi, S. A. Morin, A. A. Stokes, A. D. Mazzeo, X. Chen, M. Wang, and G. W. Whitesides, Proc. Natl. Acad. Sci. USA 108, 20400 (2011).

[4] H. Yuk, S. Lin, C. Ma, M. Takaffoli, N. X. Fang, and X. Zhao, Nat. Commun. 8, 14230 (2017).

[5] H. Lipson, Soft Robot. 1, 21 (2014).

[6] E. Acome, S. K. Mitchell, T. G. Morrissey, M. B. Emmett, C. Benjamin, M. King, M. Radakovitz, and C. Keplinger, Science 359, 61 (2018).

[7] Y.-J. Kim, S. Cheng, S. Kim, and K. Iagnemma, IEEE Trans. Robot. 30, 382 (2014).

[8] M. Calisti, M. Giorelli, G. Levy, B. Mazzolai, B. Hochner, C. Laschi, and P. Dario, Bioinspir. Biomim. 6, 036002 (2011).

[9] D. Yang, M. S. Verma, J.-H. So, B. Mosadegh, C. Keplinger, B. Lee, F. Khashai, E. Lossner, Z. Suo, and M. G. Whitesides, Adv. Mater. Technol. 1, 1600055 (2016).

[10] B. Mosadegh, P. Polygerinos, C. Keplinger, S. Wennstedt, R. F. Shepherd, U. Gupta, J. Shim, K. Bertoldi, C. J. Walsh, and G. M. Whitesides, Adv. Funct. Mater. 24, 2163 (2013).

[11] R. V. Martinez, J. L. Branch, C. R. Fish, L. Jin, R. F. Shepherd, R. M. D. Nunes, Z. Suo, and G. M. Whitesides, Adv. Mater. 25, 205 (2013).

[12] V. Cacucciolo, J. Shintake, Y. Kuwajima, S. Maeda, D. Floreano, and H. Shea, Nature (London) 572, 516 (2019).

[13] W. Wang and S.-H. Ahn, Soft Robot. 4, 379 (2017).

[14] J.-S. Koh and K.-J. Cho, IEEE/ASME Trans. Mech. 18, 419 (2013).

[15] Y. Chen, H. Zhao, J. Mao, P. Chirarattananon, E. F. Helbling, N. P. Hyun, D. R. Clarke, and R. Wood, Nature (London) 575, 324 (2019).

[16] S. Rosset and H. R. Shea, Appl. Phys. Rev. 3, 031105 (2016).

[17] C.-J. Cao, T. L. Hill, A. T. Conn, B. Li, and X. Gao, Phys. Rev. Appl. 12, 044033 (2019).

[18] T. Li, G. Li, Y. Liang, T. Cheng, J. Dai, X. Yang, B. Liu, Z. Zeng, Z. Huang, Y. Luo, T. Xie, and W. Yang, Sci. Adv. 3, e1602045 (2017).

[19] G. Gu, J. Zou, R. Zhao, X. Zhao, and X. Zhu, Sci. Robot. 3, eaat2874 (2018).

[20] X. Dong, D. Palmer, D. Axinte, and J. Kell, J. Manuf. Process. 38, 313 (2019).

[21] R. Pelrine, R. Kornbluh, Q. Pei, and J. Joseph, Science 287, 836 (2000)
[22] X. Zhao and Q. Wang, Appl. Phys. Rev. 1, 021304 (2014).

[23] M. Duduta, E. Hajiesmaili, H. Zhao, R. J. Wood, and D. R. Clarke, Proc. Natl. Acad. Sci. USA 116, 2476 (2019).

[24] E. Hajiesmaili and D. R. Clarke, Nat. Commun. 10, 183 (2019).

[25] J. Huang, S. Shian, R. M. Diebold, Z. Suo, and D. R. Clarke, Appl. Phys. Lett. 101, 122905 (2012).

[26] G. Zurlo, M. Destrade, D. DeTommasi, and G. Puglisi, Phys. Rev. Lett. 118, 078001 (2017).

[27] X. Zhao and Z. Suo, Phys. Rev. Lett. 104, 178302 (2010).

[28] J. Zhang, H. Chen, and D. Li, Phys. Rev. Appl. 6, 064012 (2016).

[29] N. Cohen and G. deBotton, Phys. Rev. Lett. 116, 208303 (2016).

[30] T. Li, C. Keplinger, R. Baumgartner, S. Bauer, W. Yang, and Z. Suo, J. Mech. Phys. Solids 61, 611 (2013).

[31] M. Giousouf and G. Kovacs, Smart Mater. Struct. 22, 104010 (2013).

[32] D. McCoul and Q. Pei, Smart Mater. Struct. 24, 105016 (2015).

[33] F. Carpi, G. Frediani, S. Turco, and D. De Rossi, Adv. Funct. Mater. 21, 4152 (2011).

[34] C. Keplinger, T. Li, R. Baumgartner, Z. Suo, and S. Bauer, Soft Matter 8, 285 (2012).

[35] F. Carpi and D. De Rossi, Mater. Sci. Eng. C 24, 555 (2004).

[36] O. P. Prabhakar, D. Sahu, and R. K. Sahu, Adv. Appl. Mech. Eng. 397 (2020).

[37] C.-H. Liu, P.-W. Lin, J.-A. Chen, Y.-T. Lee, and Y.-M. Chang, Micromachines 8, 339 (2017).

[38] H. M. Jaeger, Soft Matter 11, 12 (2015).

[39] I. Choi, N. Corson, L. Peiros, E. W. Hawkes, S. Keller, and S. Follmer, IEEE Robot. Autom. Lett. 3, 450 (2018).

[40] E. Brown, N. Rodenberg, J. Amend, A. Mozeika, E. Steltz, M. R. Zakin, H. Lipson, and H. M. Jaeger, Proc. Natl. Acad. Sci. USA 107, 18809 (2010).

[41] E. Steltz, A. Mozeika, N. Rodenberg, E. Brown, and H. M. Jaeger, in Proceedings of the IEEE/RSJ International Conference on Intelligent Robots and Systems (IROS 2009) (IEEE, Piscataway, NJ, 2009), pp. 5672-5677.

[42] T. Ranzani, G. Gerboni, M. Cianchetti, and A. Menciassi, Bioinspir. Biomim. 10, 035008 (2015).

[43] Y.-J. Kim, S. Cheng, S. Kim, and K. Iagnemma, IEEE Trans. Robot. 29, 1031 (2013).

[44] T. Lu, J. Huang, C. Jordi, G. Kovacs, R. Huang, D. R. Clarke, and Z. Suo, Soft Matter 8, 6167 (2012).

[45] Q. Pei, M. Rosenthal, S. Stanford, H. Prahlad, and R. Pelrine, Smart Mater. Struct. 13, N86 (2004).

[46] X. Zhao and Z. Suo, Appl. Phys. Lett. 93, 251902 (2008). 